\title{
Carga inmediata: situación actual
}

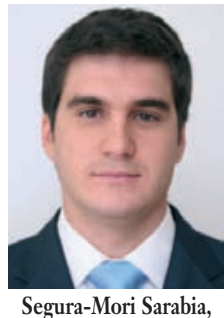

Luis

Immediate loading: curcent situation

Segura-Mori Sarabia, Luis * Díaz Vigil-Escalera, Jaime** Mauvezín Ouevedo, Mario *** González González, Innacio

* Licenciado en Odontología, Universidad Complutense de Madrid. Master en Rehabilitación Oclusal Integral. Colaborador de honor del Servicio de Prótesis y Oclusión Universidad de Oviedo.

** Licenciado en Medicina y Cirugía, Universidad de Oviedo. Licenciado Odontología UNIBE.

*** Licenciado en Odontología, Universidad de Oviedo. Colaborador de honor Servicio Prótesis y Oclusión Universidad de Oviedo.

**** Licenciado en Medicina y Cirugía, especialista en Estomatología, Universidad de Oviedo. Profesor asociado Servicio Prótesis y Oclusión Universidad de Oviedo.

\section{Correspondencia}

Luis Segura-Mori Sarabia

Servicio de Prótesis y Oclusión

Universidad de Oviedo

Facultad Odontología

E-mail: seguramori@terra.es
Resumen: En muchas ocasiones, la adopción de los protocolos clásicos de carga de los implantes no satisface las exigencias de pacientes y profesionales, por lo que la carga inmediata supone una importante línea de investigación en la Implantología moderna. Cada vez hay una mayor cantidad de publicaciones científicas referentes a esta materia, que permiten resolver un creciente número de casos de manera predecible, reduciendo al mínimo los tiempos de espera y consiguiendo nuestra satisfacción y, sobre todo, la del paciente. La estabilidad primaria, reducción de micromovimientos, microtopografía y longitud de los implantes, torque de inserción, el tipo de prótesis y la oclusión parecen ser factores determinantes en el éxito de la carga inmediata. En determinadas situaciones clínicas, como rehabilitaciones totales mandibulares, un gran número de estudios avalan resultados similares a los conseguidos con protocolos convencionales; sin embargo, y aunque es posible encontrar en la literatura tasas de éxito muy altas en casi todas las situaciones clínicas, hay algunas que requieren actuar con cautela a la espera de un mayor número de publicaciones.

Palabras clave: carga inmediata, oseointegración, estabilidad primaria, micromovimientos, oclusión.

Abstract: Many times, classical loading protocols on implants do not satisfy the expectations of both patients and clinicians. Therefore, immediate loading is supposed to be an interesting line of investigation in modern implantology. There are an important number of scientific publications available about this option, which makes it possible to solve a growing number of cases, in a predictable way, reducing waiting times and providing satisfaction to professionals and, most of all, to patients. Primary stability, reduction of micromovements, implant microtopography and length, insertion torque, prosthesis design and occlusion seem to be determinant factors for success in immediate loading. In several clinical situations, such as full mandibular restorations, a great amount of studies demonstrates similar results as the ones achieved with conventional protocols: nevertheless, although it is possible to find in the literature high success rates in most clinical situations, there are a few ones that require acting with caution until we have more publications at our disposal.

Key words: immediate loading, osseointegration, primary stability, micromovements, occlusion.

BIBLID [1138-123X (2006)11:5-6; septiembre-diciembre 497-612]

Segura-Mori Sarabia L, Díaz Vigil-Escalera J, Mauvezín Quevedo M, González González I. Carga inmediata: situación actual. RCOE 2006;11(5-6):529-541. 


\section{Introducción 4 objelivos}

Cuando Bränemark' introdujo el concepto de Osteointegración en 1969 se estableció un protocolo de carga de los implantes, que debía realizarse tras 6 meses en maxilar y 4 en mandíbula para lograr una adecuada cicatrización ósea y remodelación de la interfase hueso-implante, sin interposición de tejido fibroso. El hecho de adoptar estos protocolos en la Implantología clínica supone unas tasas de éxito muy elevadas, por encima del $95 \% \%^{1.15}$, con una predecibilidad casi exacta en la evolución del caso. Sin embargo, la creciente exigencia de los profesionales $y$, sobre todo, de los pacientes, hace que estos largos tiempos de espera sean inaceptables en muchos casos. Por ello, casi todas las líneas de investigación en la Implantología moderna van dirigidas a la reducción o eliminación de los tiempos de espera entre la fase quirúrgica y protésica. Así, se están llevando a cabo numerosos estudios histológicos que permiten conocer con exactitud los mecanismos que acontecen en la osteointegración y así poder intervenir en ellos para acortar sus tiempos. Además, se están estableciendo nuevos protocolos quirúrgicos y protésicos, mientras que las casas comerciales están desarrollando infinidad de superficies y diseños de implantes con este mismo fin.

El objetivo del presente trabajo es hacer una revisión bibliográfica entre los años 1979-1999 y, sobre todo, 2000-2006 de las publicaciones científicas de mayor impacto en relación a la carga inmediata de implantes dentales, para determinar y comparar:

- protocolos quirúrgicos y protésicos

- ventajas e inconvenientes

- factores de influencia

- factores de riesgo

- criterios de inclusión-exclusión

- criterios de éxito-fracaso

- resultados

- conclusiones

\section{Material प metodología}

Se consultaron los artículos en la materia en la base de datos de PubMed, libros, ponencias en congresos y publicaciones en revistas españolas. Se aplicó criterio de inclusión a los artículos que estudiaban al menos 50 implantes de carga inmediata y con un seguimiento mínimo de 1 año. No se excluyeron las publicaciones que no fueron elaboradas bajo estricta metodología para la investigación. Entre las revistas consultadas se encuentran Internacional Journal Periodontics Restorative Dentistry, Internacional Journal of Oral and Maxillofacial Implants, Internacional Journal of Prosthodontics, Clinical Oral Implant Research, Journal of Periodontology, Periodontology 2000, Journal of Oral and Maxillofacial Surgery y Clinical Implant Dentistry and Related Research.

\section{Concepto, antecedentes y estado actual}

Cuando hablamos de carga inmediata nos referimos a la colocación de la prótesis antes de las 48 horas tras la colocación del implante ${ }^{3,459}$, aunque hay autores que prolongan este tiempo hasta los diez días². Es importante diferenciar el concepto de carga oclusal inmediata o carga funcional inmediata, del concepto de carga no funcional inmediata o carga estética. El primero hace referencia a la colocación de la prótesis sometida a fuerzas oclusales normales para ese paciente y que se transmiten directamente sobre el implante/pilar 5-10,13,76, mientras que en la carga estética la restauración se deja en mínima oclusión o anoclusión ${ }^{11-13,76}$.

\section{Antecedentes (1979-99)}

Ledermann ${ }^{14}$ fue el primero en publicar un estudio de implantes sometidos a carga inmediata. Utilizó fijaciones de superficie TPS, colocados a nivel intermentoniano, con anclaje bicortical y que soportarían una sobredentadura. Hizo un seguimiento a 81 meses de 476 implantes obteniendo una tasa de éxito del 91,2\%.

Esto ocurría en 1979, y a partir de ahí comienzan a publicarse artículos sobre la materia de manera progresiva. Así, en 1983 Schroeder, quién había introducido el concepto de anquilosis funcional18, publica un estudio de 4 implantes de función inmediata con 17 meses de seguimiento, en el que, con el mismo tipo de pacientes que Ledermann, consigue un $98,1 \%$ de éxito ${ }^{17}$.

En 1986 Babbush y cols. ${ }^{19}$, colocan 514 implantes TPS en región sinfisaria 
cargados con sobredentaduras con barra dolder, y tras un seguimiento de más de 5 años logran un éxito del $96,1 \%$.

Dietrich y cols. ${ }^{20}$ publican en 1993 un trabajo en el que, tras cargar de forma inmediata 421 implantes IMZ, obtienen un $92,5 \%$ de éxito a los 6 meses y un $86,3 \%$ a los 5 años.

Cuatro años más tarde, Chiapasco y cols. ${ }^{21}$ presentan un amplio estudio retrospectivo de 904 implantes (380 de superficie TPS, 152 ITI, 208 Ha-Ti y 164 NLS) sometidos a carga inmediata. Colocaban cuatro fijaciones de al menos $10 \mathrm{~mm}$ de longitud y con anclaje bicortical en sínfisis mandibular para cargarlos con una sobredentadura sobre barra de oro. Realizaron un seguimiento de 194 de los 226 pacientes tratados que variaba entre los 2, 4, 5, 69 y 13 años, logrando un $96,9 \%$ de éxito, sin diferencias estadísticamente significativas entre los diferentes tipos de implantes.

Ese mismo año Tarnow y cols. ${ }^{22}$ dan a conocer los resultados de un estudio de 107 implantes Nobel, ITI, $3 i$ y Astra 69 sometidos a carga inmediata y 38 retardada) colocados sobre 10 pacientes totalmente edéntulos $(6$ de ellos intervenidos en mandíbula y 4 en el maxilar). Ponían un mínimo de 10 fijaciones de al menos $10 \mathrm{~mm}$ por arcada, dejando al menos 5 de ellos sumergidos por motivo médico-legal; el resto se cargaba con una prótesis fija provisional el mismo día de la cirugía. Tras un seguimiento de 12 a 60 meses obtuvieron un éxito del $97,1 \%$ en los de carga inmediata y un $97,4 \%$ en los de carga retardada.

También en 1997, Balshi y Wolfinger ${ }^{23}$ publican un estudio sobre 13 pacientes en el que colocan 10 implantes Bränemark de al menos 7 $\mathrm{mm}$ en la mandíbula de cada uno de ellos. De estos implantes, 40 (4 por paciente) se cargan tras la cirugía para soportar una prótesis transicional fija. Con este protocolo tan sólo consiguen un $80 \%$ de éxito, mientras que esta tasa se eleva al 95,6\% en los cargados según protocolo convencional.

Scortecci ${ }^{24}$ logra un año después un $98 \%$ de tasa de éxito en un estudio de 6 a 48 meses de seguimiento de 783 implantes de disco de inserción lateral colocados en maxilares con atrofia moderada-severa y cargados con prótesis fija inmediata. Utilizó radiografías, análisis clínicos, periotest y control de torque a $20 \mathrm{Ncm}$ como criterios de éxito.

\section{Situación actual (2000-2006)}

En el año 2000, Horiuchi y cols. ${ }^{25}$ publican los resultados de un estudio de 140 implantes de carga inmediata con seguimiento de 8 a 24 meses. Se intervinieron 12 mandíbulas y 5 maxilares, en los que se colocaron implantes Bränemark con un torque de inserción de al menos $40 \mathrm{Ncm}$ sobre los que se colocaba una prótesis fija provisional inmediata de resina con un refuerzo interno de cromo-cobalto. Consiguieron un $95,5 \%$ de éxito en el maxilar y un $97,9 \%$ en la mandíbula.

Ese mismo año, Gatti y cols. ${ }^{26}$ obtienen un $96 \%$ de éxito tras un estudio retrospectivo con seguimiento de 25 a 60 meses en el que colocaron 84 implantes ITI intermentonianos en 21 pacientes (4 por paciente) cargados con una sobredentadura inmediata sobre barra de oro.

También en 2000, Jaffin y cols. ${ }^{27}$ publican un estudio en el que coloca- ron 149 implantes ITI de superficies TPS y SLA de al menos $10 \mathrm{~mm}$ de longitud, en 27 mandíbulas (donde se ponían al menos 4 implantes) y 4 maxilares (al menos 6 fijaciones). Los cargaban 72 horas tras la cirugía con prótesis fija de metal-acrílico. Tras un seguimiento de entre 6 y 60 meses lograron un $97 \%$ de éxito.

De nuevo en este año, Maló y cols. ${ }^{28}$ presentan un trabajo sobre 94 implantes de carga inmediata en zona estética de ambos maxilares, buscando anclaje bicortical y con un torque de inserción de al menos $32 \mathrm{Ncm}$. En cuanto a los aspectos protéticos, utilizaban prótesis fijas implantorretenidas: 23 puentes (14 maxilares y 9 mandibulares) y 31 coronas (22 maxilares y 9 mandibulares). Estas restauraciones quedaban en anoclusión y los pacientes eran instruídos para no morder en esa zona. Tras un seguimiento de 1-2 años, obtuvieron una tasa de éxito del $96 \%$. Todos los fracasos se produjeron en zonas de reciente extracción.

En 2001, Ganeles y cols. ${ }^{29}$ dan a conocer un estudio clínico sobre 161 implantes con un seguimiento de entre 13 y 41 meses. Tras colocar entre 5 y 8 implantes por paciente con diferentes tipos de desdentación, los cargaban de forma inmediata con prótesis fija de diferentes diseños, cementados y atornillados. Lograron un éxito del 99,3\%.

Ese mismo año Glauser y cols. ${ }^{30}$ publican un estudio prospectivo a 1 año de implantes Bränemark de carga inmediata. Colocaron un total de 127 fijaciones (76 maxilares y 51 mandibulares) sobre 41 pacientes. Al $71 \%$ de los mismos se les colocó la prótesis el mismo día, al resto dentro de los 11 
días postquirúrgicos. Respecto a los aspectos oclusales, todas las prótesis tenían contactos normales en relación céntrica, y no fueron excluidos los bruxistas. Tuvieron una tasa de éxito del $82,7 \%$ (91\% de éxito si excluimos el maxilar posterior; $66 \%$ de éxito en esta región). Añaden que en bruxómanos se produjo un fracaso del $41 \%$ de los implantes en comparación con el $12 \%$ de los no bruxómanos. También apuntan una tasa de éxito considerablemente superior en implantes asociados a regeneración ósea guiada (90\% frente a un $67 \%$ ).

Un año más tarde Ibáñez y cols. ${ }^{31}$ obtienen una tasa de éxito del 100\% tras un seguimiento de 2-3 años en un estudio de 87 implantes Osseotite de $3 i$ sobre 11 pacientes, cargados de forma inmediata, tanto maxilar como mandíbula, con prótesis fija provisional.

Ese mismo año, May y Romanos ${ }^{32}$ dan a conocer los resultados de su trabajo donde, tras la carga inmediata de 204 implantes Ankylos de ente 11 y $17 \mathrm{~mm}$ en región intermentoniana mediante sobredentadura, y con un seguimiento de casi año y medio, logran un $97,5 \%$ de éxito.

También en 2002, los españoles PiUrgell y Vericat-Queralt ${ }^{33}$ presentan los resultados de un estudio de implantes de carga inmediata siguiendo el protocolo estandarizado de Bränemark Novum, en el que colocan 3 implantes intermentonianos de $5 \times 11,5 \mathrm{~mm}$ sobre 25 pacientes, lo que hace un total de 75 implantes cargados. Sobre ellos colocan una prótesis fija atornillada definitiva, y tras un seguimiento de entre 6 meses y 2 años, consiguen una tasa de éxito del $92 \%$.

Un año más tarde Callandriello y cols. ${ }^{34}$ publican un estudio sobre 50 implantes Branëmark de superficie maquinada de carga inmediata, colocados mayoritariamente en región posterior de ambas arcadas (78\% de la fijaciones), con un torque de inserción de 40-72 Ncm. Cargaban los implantes con prótesis provisionales fijas con oclusión ligera, y tras un año de seguimiento refieren un $98 \%$ de éxito.

Rocci y cols. ${ }^{35}$ dan a conocer los resultados de un estudio de seguimiento a 3 años de 97 implantes Bränemark MkIV maquinados colocados en maxilar superior siguiendo un protocolo de cirugía sin colgajo, colocación de las fijaciones en lugar predeterminado y cargados con una prótesis fija prefabricada ( 25 parciales y 27 unitarias). Logran un $97 \%$ de éxito, siendo los fracasos antes de las ocho semanas y en fumadores, hueso blando o implantes unitarios.

También en 2003, Testori y cols. ${ }^{41}$ relatan un $98,9 \%$ de éxito de 92 implantes Osseotite en mandíbula sobre 14 pacientes cargados 36 horas tras la cirugía mediante prótesis híbridas.

Ese mismo año, Glauser y cols. ${ }^{45}$ rehabilitan a 38 pacientes con 102 implantes Bränemark MkIV TiUnite (38 maxilares y 64 mandibulares). De un total de 51 rehabilitaciones, 20 eran unitarias, 30 parciales y una de arcada completa. El $88 \%$ de las fijaciones se colocaron en sector posterior y el $76 \%$ en hueso blando. Tras cuatro años de seguimiento lograron un $97,1 \%$ de éxito, con una pérdida ósea periimplantaria de 1,2 $\pm 0,9 \mathrm{~mm}$.

Paulo Maló y cols. ${ }^{52}$ introducen en 2003 el concepto "All-on-Four» con implantes Bränemark de carga inme- diata. Este protocolo consiste en la colocación de 4 implantes intermentonianos de al menos $10 \mathrm{~mm}$, los dos anteriores en la dirección del hueso y los dos posteriores con una inclinación de unos $30^{\circ}$, emergiendo a nivel del segundo premolar. La prótesis híbrida que soportan los implantes, previa colocación de pilares angulados en las fijaciones posteriores, reponen la arcada hasta el primer molar, y eran colocadas antes de dos horas tras la cirugía. En el trabajo publicado, sobre 44 pacientes, colocaron 176 implantes de carga inmediata (de los cuales 45 eran inmediatos) y otros 62 implantes de rescate. Tras un seguimiento de entre 6 meses y 2 años, logran un éxito del $96,7 \%$ y un $100 \%$ de la prótesis.

Este mismo autor publica también en 2003 un trabajo de 116 implantes Bränemark (de los que 22 eran inmediatos) de carga inmediata en zona estética maxilo-mandibular. Colocaron 74 implantes en maxilar y 42 en mandíbula sobre 66 pacientes (entre los que había hipertensos, asmáticos, fumadores, bruxistas y medicados por patología hepática, cardiaca y respiratoria). Las fijaciones debían ser de al menos $10 \mathrm{~mm}$ y lograr una buena estabilidad primaria. Se rehabilitó con 63 coronas unitarias y 24 puentes, dejándolos en anoclusión y se instruyó para no masticar con ellos. Tras un seguimiento de 1 año, logaron un $95,7 \%$ de éxito $193,7 \%$ en unitarios y $98,1 \%$ en ferulizados) $)^{53}$.

Ya en 2004, Testori y cols. ${ }^{62}$ consiguen un $97,4 \%$ de éxito en un estudio de seguimiento de 8-65 meses, sobre116 implantes 3i Osseotite de carga inmediata. Colocaban $5 \circ 6$ implantes en cada uno de los 16 
pacientes tratados, para cargarlos con una prótesis híbrida inferior.

Jaffin y cols. ${ }^{64}$ realizan en 2004 un estudio en el que rehabilitan 43 pacientes con edentulismo completo maxilar con 6-8 implantes de al menos $8 \mathrm{~mm}$. Colocaron un total de 236 implantes cargados entre 48 y 72 horas tras la cirugía. Lograron un 93\% de éxito, atribuyendo los fracasos a micromovimientos producidos por masticación dura.

Ese mismo año, Drago y Lazarra65 dan a conocer los resultados de un estudio sobre 38 pacientes parcialmente edéntulos a los que colocan 93 implantes Osseotite de 3i. Realizan carga inmediata mediante prótesis provisional cementada sin contactos céntricos ni excéntricos. Tras un seguimiento de al menos 18 meses consiguen un 97,4\% de éxito, habiendo diferencias estadísticamente significativas entre implantes ferulizados y unitarios (que reciben mayor carga lateral).

También en 2004, Galluci y cols. ${ }^{66}$ presentan un trabajo sobre 8 pacientes con edentulismo total en arcada superior, inferior 0 ambas. Rehabilitaron un total de 11 arcadas, colocando entre 6 y 10 implantes en cada una de ellas, lo que hace un total de 78 fijaciones. Realizaron un encerado diagnóstico previo, sobre el que confeccionarían las prótesis completas provisionales totalmente acrílicas antes a la cirugía. El mismo día de la intervención, se atornillaba la misma, y tras 8 20 de seguimiento logran un $97,4 \%$ de éxito, siendo los fracasos dos implantes de $8 \mathrm{~mm}$.

Callandriello y Tomatis $^{108}$ realizan ese mismo año un interesante estudio de 98 implantes unitarios Bränemark de al menos $10 \mathrm{~mm}$ y con un torque de inserción de al menos $20 \mathrm{Ncm}$. Estas fijaciones eran colocadas principalmente en región posterior de ambos maxilares. Consiguen un $98,9 \%$ de éxito tras un año de seguimiento.

Un año más tarde, Balshi y cols. ${ }^{70}$ publican un estudio de 522 implantes Bränemark para rehabilitación inmediata de maxilares totalmente edéntulos. Colocaron al menos 10 implantes por arcada, cargados con prótesis provisional acrílica atornillada en el momento de la cirugía; logaron un 99\% de supervivencia de los implantes y un $100 \%$ en la prótesis.

También en 2005, Martínez y cols. ${ }^{69}$ logran un $100 \%$ de éxito en un trabajo de 80 implantes Defcon TSA Avantblast de $13 \mathrm{~mm}$ colocados de cuatro en cuatro en mandíbulas para recibir una sobredentadura inmediata con dos caballitos, 48 horas postcirugía.

Este mismo año, Degidi y cols. ${ }^{73}$ presentan un estudio de seguimiento a 7 años sobre 93 implantes de carga inmediata. Rehabilitaron 7 arcadas completas y 9 parciales, tanto maxilares como mandibulares, mediante sobredentaduras, prótesis acrílica provisional o puentes definitivos de metal-cerámica, durante las 24 horas siguientes a la colocación de los implantes. Tuvieron 6 fracasos que ocurrieron durante el primer año; de los cuales 4 eran prótesis parciales y 3 en hueso tipo III. Esto supuso una tasa de éxito del $93,5 \%$ a los 7 años. La pérdida ósea media fue de 0,6 $\mathrm{mm}$ tras el primer año y de 1,1 mm tras 7 .

Aalam y cols..$^{71}$ colocaron un total de 90 implantes Bränemark MkIII sobre 16 pacientes para soportar una prótesis híbrida inferior inmediata. Consiguieron un $96,6 \%$ de éxito a los 3 años con una pérdida ósea de 1,2 $\pm 0,1 \mathrm{~mm}$.
Todavía en 2005, Degidi y cols. ${ }^{77}$ presentan un estudio, con seguimiento a 5 años, de 388 implantes colocados en maxilares edéntulos. Rehabilitaron mediante herraduras provisionales acrílicas al momento de la cirugía consiguiendo un 98\% de éxito.

Ibáñez y cols. ${ }^{76}$ publican en 2005 un estudio de 343 implantes 31 Osseotite sobre 41 pacientes (incluyendo fumadores y bruxistas) para rehabilitación completa de 26 maxilares y 23 mandíbulas. Antes de 48 horas tras la colocación de los implantes, éstos eran cargados mediante prótesis definitiva metal-porcelana o metalacrílico; o prótesis provisional acrílica o metal-acrílica. En cuanto a los aspectos oclusales, buscaban contactos bilaterales en relación céntrica y guía canina. Tras un seguimiento de 12 a 74 meses refieren un $99,42 \%$ de éxito.

Östman y cols. ${ }^{89}$ colocan 123 implantes Nobel TiUnite sobre 20 pacientes con maxilar totalmente edéntulo. Tras 12 horas, cargaron los implantes con un puente provisional atornillado. Compararon los resultados con un grupo de otros 20 pacientes sobre los que pusieron 120 implantes cargados mediante protocolo clásico. Lograron un 99,2 \% de éxito en los implantes de carga inmediata y un $100 \%$ en los de retardada.

Ormianer y cols..$^{93}$ publican en marzo del presente año el primer estudio de implantes cargados de forma inmediata mediante sobredentaduras inferiores retenidas por bolas. En una muestra de 14 pacientes colocaron 2 implantes en cada uno de ellos. Lograron un $96,4 \%$ de éxito.

En junio de 2006, Lindeboom y cols. ${ }^{96}$ comparan en un estudio los 
resultados de 50 implantes Biocomp TPS. A todos los implantes se les colocaron provisionales 24 horas postcirugía, la mitad de ellos recibieron contactos normales en relación céntrica y movimientos excursivos; mientras que en los otros 25 implantes se eliminaron los contactos céntricos y excéntricos. Se recomendó dieta blanda en todos los casos durante 6 semanas. Fracasaron 2 implantes de carga inmediata y 3 de carga diferida. Se consiguió mayor regeneración de la papila mesial en los implantes de carga diferida, siendo igual en los dos grupos la regeneración de la distal. El trabajo concluye que no hay diferencias estadísticamente significativas entre ambos grupos.

Degidi y cols. ${ }^{99}$ hacen también un estudio comparativo de 1005 implantes Dentsplay-Friadent Xive sobre 371 pacientes. De ellos 484 se cargaron de forma inmediata y 521 diferida. Los resultados mostraron un $98,7 \%$ de éxito en el primer grupo y un $99,4 \%$ en el segundo.

Klee de Vasconcellos y cols. ${ }^{100}$ presentaron en julio de 2006 un protocolo para rehabilitación de mandíbulas totalmente edéntulas mediante prótesis híbrida inmediata. El protocolo "Speed Master» consiste en la colocación de 4 implantes intermentonianos mediante una guía del sistema sobre la que se coloca una prótesis fija definitiva con barra de titanio prefabricada. El estudio se realizó sobre 15 pacientes (lo que hace un total de 60 implantes), y tras un seguimiento de 15 a 27 meses se logró un 100\% de éxito con una pérdida ósea media de $1,11 \mathrm{~mm}$.

RCOE, 2006, Vol 11, N05-6, 529-541

\section{Aspectos a considerar}

\section{Criterios de éxito-fracaso}

Albrektsson y cols. ${ }^{106,107}$ publican en 1986 y 1991 sendos artículos en los que enumera los signos y síntomas clínicos que determinan el éxito de la oseointegración de los implantes:

- Inmovilidad del implante testado clínicamente.

- No evidencia radiográfica de radiolucidez periimplantaria

- La pérdida ósea anual no debe ser mayor de 1,5-2 $\mathrm{mm}$ el primer año y

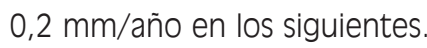

- Ausencia de signos y síntomas como el dolor, inflamación severa, infección, neuropatías, hiperestesias o invasión del conducto dentario.

\section{Ventajas e inconvenientes de la carga inmediata}

- Tiempo: probablemente esta sea la principal ventaja de la carga inmediata de los implantes, se reduce entre unos tres y seis meses el tiempo transcurrido desde la colocación de las fijaciones hasta su puesta en función ${ }^{21,26,35,53,65,79,100}$.

- Satisfacción del paciente: el edentulismo total o parcial supone en la mayoría de los casos una afectación psicológica del paciente ${ }^{21,28}$. Por otra parte, la inestabilidad de las prótesis transicionales convencionales suele resultar incómoda ${ }^{21}$, por lo que la colocación de una prótesis inmediata implantosoportada supone un gran confort y satisfacción para el pacien$\mathrm{te}^{26,36,53}$.

- Economía: son varios los autores que consideran que la carga inmediata no supone un coste adicional para el paciente 0 incluso resulta más económico ${ }^{12,30,52}$; sin embargo, otros auto- res como Attard y cols. ${ }^{90}$ aseguran que el protocolo de carga inmediata no abarata costes para el paciente. Probablemente los costes estén en función de si la carga inmediata se realiza con la prótesis definitiva, como en el protocolo All-on-Four ${ }^{52}$, o si es necesaria una prótesis provisional.

- Riesgo: el hecho de cargar los implantes sin haberse producido la oseointegración determina una evidente situación de riesgo añadido.

\section{Factores de influencia para el éxito en carga inmediata}

\section{Estabilidad primaria}

En la actualidad se define la estabilidad primaria como la ausencia de movimiento del implante tras la inserción del mismo ${ }^{13}$. Este hecho es un requisito indispensable para el éxito de la oseointegración de los implantes de carga inmediata $7,12,13,42,53-55,59,73,94,95,108$. La estabilidad primaria puede medirse mediante un sencillo test de movilidad manual, mediante la magnitud de torque de inserción, el uso del Periotest (mide la amortiguación de los tejidos periimplantarios tras aplicar fuerza axial ligera) o la utilización del Ostell, que mide la frecuencia de resonancia de un piezoeléctrico unido al implante tras estimulación de una pequeña corriente eléctrica ${ }^{13}$, en valores ISQ, cuyo valor ha de ser entre 60 y 65 para la carga inmediata ${ }^{110}$.

\section{Minimización de micromovimientos}

Tan importante como la estabilidad primaria es mantener la inmovilidad del implante durante el período de cicatrización de los tejidos periimplantarios $^{94}$, para evitar la formación de 
tejido fibroso en la interfase hueso implante ${ }^{80}$, y por lo tanto fracaso del implante ${ }^{64}$. Kawahara y cols. ${ }^{43}$ consideran que la magnitud de los micromovimientos inferior a 30 micras no influye negativamente en la osteogénesis y crecimiento óseo alrededor del implante. Por su parte, Ibáñez y cols. ${ }^{74}$ toleran en su estudio una magnitud de unas 100 micras. Duyck y cols.91 refieren la mayor creación de volumen óseo en implantes de carga inmediata en ausencia de micromovimientos o con micromovimientos de 90 micras; así como una mayor densidad ósea cuando las fijaciones sufren micromovimientos de 60-90 micras. Lekholm ${ }^{104}$ recomienda no retirar la rehabilitación protésica durante el periodo cicatricial iniacial para evitar los micromovimientos.

\section{Supeficie del implante}

La microtopografía del implante desempeña un papel fundamental en el proceso de integración ósea ${ }^{42,47,5,5,59,69,76,98}$, ya que puede aumentar significativamente el contacto hueso-implante, incrementando la fuerza de adhesión al hueso, además de facilitar un crecimiento más rápido. Por lo tanto, se puede afirmar que las superficies rugosas (que aumentan significativamente la superficie del implante) pueden promover la osteoconducción, incrementando la superficie para la fijación de la fibrina y proporcionando características en su superficie en las que se puede enredar la fibrina. Por otra parte, la química de algunas superficies del implante pueden aumentar la absorción y retención de macromoléculas del medio biológico, potenciándose también la osteoconducción ${ }^{103}$. Albrektsson y cols. ${ }^{85,86}$ concluyen en un

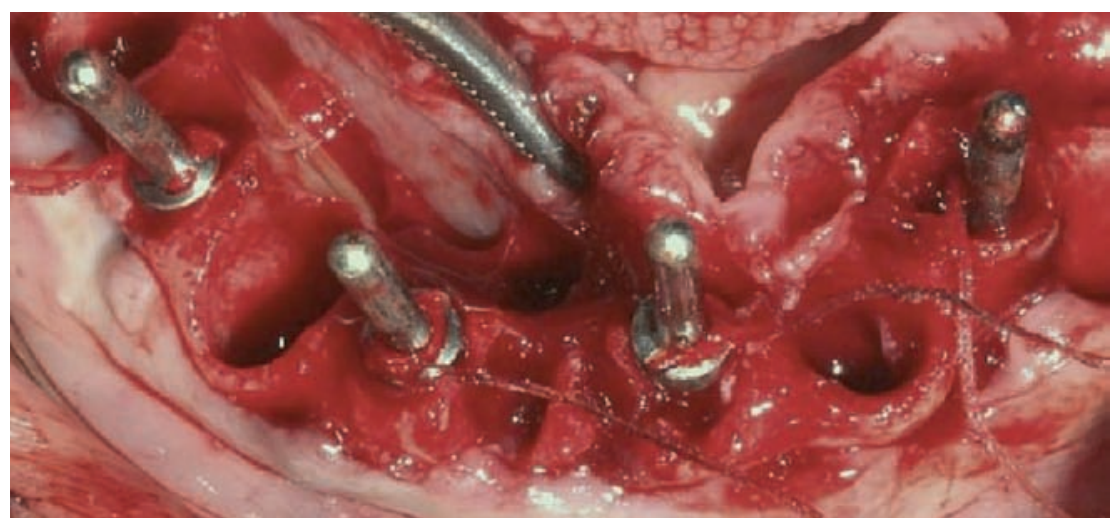

Figura 1. Indicadores de dirección tras fresado de los lechos postextracción.

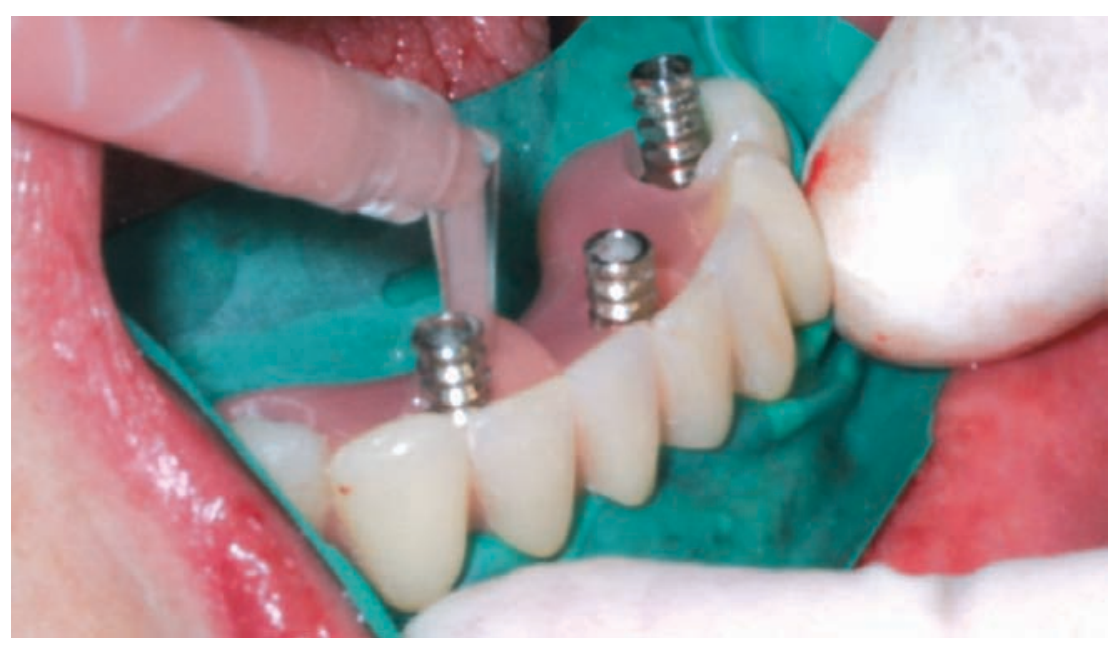

Figura 2. Tras la colocación de los cilindros provisionales y aislar con dique de goma, se rellenan las chimeneas con resina autopolimerizable.

estudio que las superficies moderadamente rugosas presentan ligeras ventajas que las maquinadas o más rugosas. Sul y cols. ${ }^{97,101}$ publican en 2006 dos estudios en los que estudian las propiedades de un implante experimental con superficie de magnesio oxidado, concluyendo que esta superficie aumenta la neoformación ósea y agiliza la oseointegración.

\section{Calidad-cantidad ósea}

La presencia de un volumen adecuado de hueso para alojar los implantes y, sobre todo, una buena calidad del mismo ${ }^{104}$, también parece ser un factor importante en los implantes de carga inmediata42,44,58,59,87. Así, Misch y cols. ${ }^{49}$ consideran que en el hueso tipo IV es 10 veces más débil que el tipo I. Rocci y cols. ${ }^{35,47}$ asocian el fracaso de varios implantes, entre otras causas, a la colocación de los mismos en hueso blando. Vanden Boagaerde y cols. ${ }^{54}$ afirman que en zonas de baja densidad ósea es fundamental el logro de una gran estabilidad primaria para conseguir éxito. 
Sin embargo, Ibáñez y cols. ${ }^{76}$ consideran que la calidad ósea no es tan importante como se creía, especialmente al utilizar implantes de superficie rugosa. Por su parte, Clauser y cols. ${ }^{30}$ consideran que los defectos óseos son un factor de riesgo importante. (fig. 1 y 2).

\section{Torque de inserción}

Este aspecto es también de suma importancia para el éxito en carga inmediata, y está directamente relacionado con el apartado anterior. Para Maló y cols. ${ }^{53}$ y Drago y cols. ${ }^{95}$ el torque de inserción mínimo ha de ser de $30 \mathrm{Ncm}$. Neugebauer y cols. ${ }^{94}$ consideran que éste ha de ser de $35 \mathrm{Ncm}$, mientras que Hui ${ }^{12}$, Horiuchi ${ }^{25}$ y Calandriello ${ }^{34}$ aumentan el torque de inserción mínimo para el éxito en carga inmediata a $40 \mathrm{Ncm}$.

\section{Oclusión}

La dirección y magnitud de las fuerzas oclusales también juegan un papel importante en la carga inmediata ${ }^{42,59,61,69}$. Algunos autores recomiendan eliminar todo contacto en todos los movimientos ${ }^{12,28}$. Otros como Calandriello y cols. ${ }^{7,34,108}$ permiten una oclusión suave. Clauser y cols. ${ }^{30,96} \mathrm{e}$ Ibáñez y cols. ${ }^{76}$ le dan contacto normal en relación céntrica y movimientos excursivos a sus prótesis sobre implantes de carga inmediata. Tsirlis ${ }^{72}$ considera que en zona estética es recomendable la carga no funcional para conseguir buena estabilidad primaria y minimizar al máximo la pérdida ósea. Isidor ${ }^{105}$ defiende que una sobrecarga masticatoria con buena higiene periimplantaria conlleva pérdidas óseas frente a una higiene defectuosa sin sobrecargas en las que

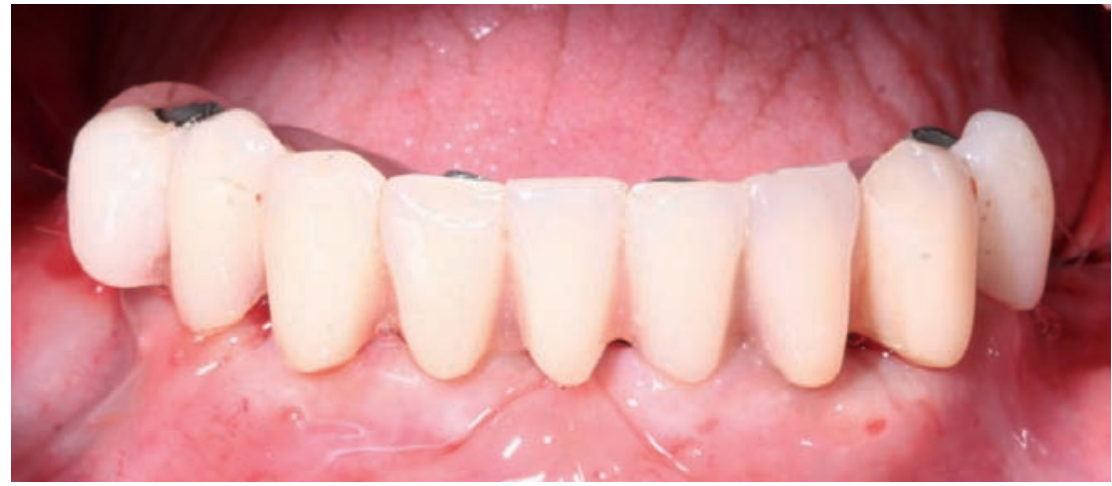

Figura 3. Aspecto en boca de una prótesis completa inferior implantosoportada provisional de carga inmediata.

no se produce esta pérdida. En cuanto a la masticación, hay varios autores que recomiendan dieta blanda durante el período de cicatrización ${ }^{46,61,96}$.

\section{Paciente: hábitos}

Ciertos hábitos de los pacientes tienen un efecto nocivo en la oseointegración de implantes de carga inmediata ${ }^{44,49,59}$, siendo los principales el tabaquismo y los hábitos parafuncionales El tabaquismo supone un factor de riesgo en el éxito de los implantes en general. Rocci y cols. 35.47 atribuyen al tabaco (entre otras causas) el fracaso de la oseointegración de varios implantes de carga inmediata. Como promedio, se pierden el doble de implantes en fumadores que en no fumadores, por lo que el consumo de tabaco debe considerarse una contraindicación relativa para la carga inmediata ${ }^{104}$. Sin embargo, varios autores como Ganeles y cols. ${ }^{20} \mathrm{e}$ Ibáñez y cols. ${ }^{76}$ incluyen fumadores en sus estudios sin que éstos reporten resultados negativos.

En cuanto a los hábitos parafuncionales, un gran número de estudios excluyen a los pacientes bruxis$\operatorname{tas}^{21,28,53,54,73}$ y otros que los incluyen refieren tasas de fracaso mayores que en pacientes no bruxistas ${ }^{30}$. Sin embargo, otro trabajos no excluyen a estos pacientes ${ }^{53}$ y logran buenos resultados, aunque Ibáñez y cols. ${ }^{76}$ refieren que los dos únicos implantes fracasados en su estudio fueron colocados en bruxistas. El bruxismo también se debería considerar una contraindicación relativa ${ }^{104}$

\section{Paciente: factores locales y sisté- micos}

Lekholm ${ }^{104}$ publica en 2003 un estudio de carga inmediata y temprana en pacientes de riesgo. En cuanto a los factores de riesgo sistémicos, aparte del tabaquismo ya comentado, el autor considera que la edad y el sexo no representan un problema en cuanto a los protocolos de carga inmediata, aunque recuerda que en pacientes de edad avanzada hay mayor riesgo de complicaciones y la cicatrización es más lenta; también recomienda evitar la colocación de implantes en pacientes en crecimiento. Tampoco considera la diabetes una situación de riesgo potencial. No así en pacientes con raquitismo (contraindicación absoluta), osteoporosis 
o síndrome de Sjögren, considerados potencialmente de riesgo. En relación a los factores de riesgo locales, aparte de la calidad-cantidad ósea y el bruxismo ya citados, los pacientes no colaboradores y las zonas de injerto óseo son considerados factores de riesgo. Los pacientes con maxilares irradiados han de considerarse una contraindicación absoluta.

\section{Longitud, diámetro y número de los implantes}

Estos factores tienen también mucha influencia para lograr resultados positivos en carga inmediata ${ }^{42,44,49}$. Parece haber bastante consenso en la longitud mínima de los implantes de carga inmediata, siendo ésta de 10 $\mathrm{mm}^{21,22,52,53,104}$. Algunos autores como Jaffin y cols. ${ }^{64}$ y Gallucci y cols. ${ }^{66}$ reducen la longitud mínima de los implantes a $8 \mathrm{~mm}$; Balshi y cols. ${ }^{23}$ llegan a colocar implantes de $7 \mathrm{~mm}$ para carga inmediata. En cuanto al diámetro de los implantes no hay demasiada información publicada; la mayoría de los autores que hacen referencia a este dato emplean fijaciones de entre 3,3 y $4 \mathrm{~mm}^{21,52,53}$. Lekholm ${ }^{104}$ afirma que cuanto más largos y mayor diámetro tengan los implantes, mejores resultados se obtendrán, sin embargo, Degidi y cols. ${ }^{77}$ consideran que los implantes de mayor diámetro suponen un mayor riesgo de fracaso. Misch y cols. ${ }^{49}$ recomiendan la colocación de más implantes y más largos para conseguir mejores resultados.

\section{Tipo de prótesis}

Según los trabajos revisados, el tipo de prótesis juega un papel importante en los resultados de los implantes cargados de forma inmediata. Amplia-

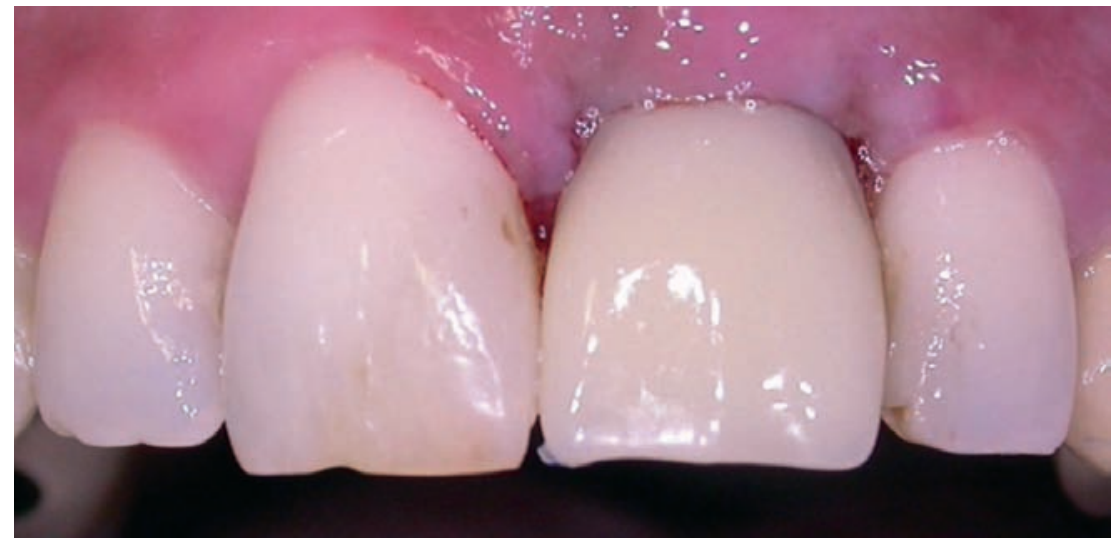

Figura 4. Imagen intraoral de una restauración unitaria provisional inmediata en el 2.1.

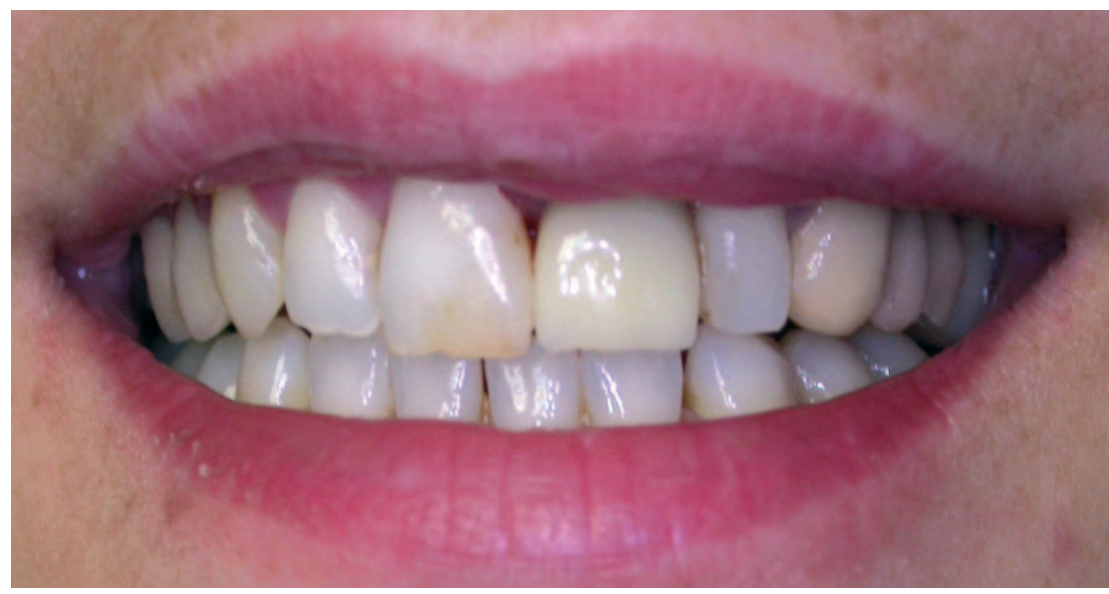

Figura 5. Imagen extraoral de la restauración anterior

mente demostrado está el éxito de las sobredentaduras y prótesis híbridas inferiores, con resultados similares a los de la carga según protocolos convencionales ${ }^{6,19,21,26,32,36,52,62,69,78,79,81}$ (Fig. 1, 2 y 3). No hay diferencias significativas en los resultados que se consiguen mediante prótesis fija provisional 0 definitiva, atornillada o cementada ya sean unitarias ${ }^{12,28,35,45}$, parciales ${ }^{7,27,28,35,45,47}$ o completas maxilares, ${ }^{9,25,27,70}$ (Fig. 4, 5 y 6). Un concepto que parece muy importante para lograr la oseointegración en carga inmediata es la ferulización de los implantes mediante la pró- tesis, ya que hay una mejor distribución de las fuerzas minimizando los micromovimientos ${ }^{6,7,22,53,58,65,104}$. Sin embargo, Abboud y cols. ${ }^{75}$ concluyen un estudio afirmando que la carga inmediata de implantes no ferulizados en sector posterior puede ser un tratamiento viable. Para favorecer la ferulización de los implantes, reduciendo las cargas nocivas sobre los mismos, muchos autores aportan rigidez a la prótesis mediante estructuras metálicas $^{25,27,31,35}$. Por otro lado, Misch y cols. ${ }^{61}$ y Lekholm ${ }^{104}$ recomiendan evitar los cantilevers. 


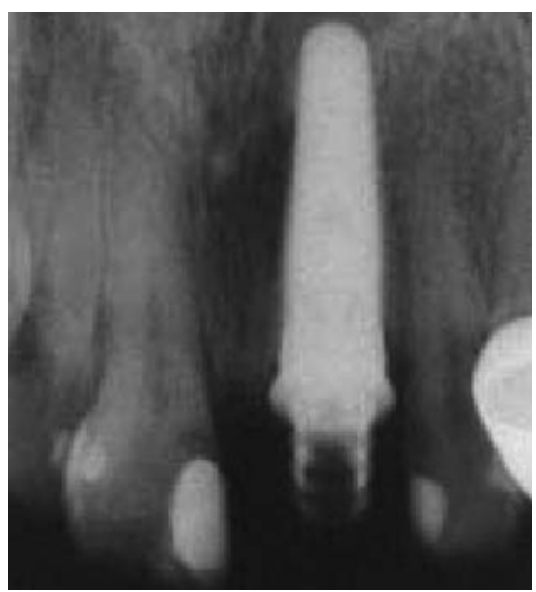

Figura 6. Imagen radiográfica del implante con restauración unitaria en carga inmediata..

\section{Histología en carga inme- diata}

Son numerosos los estudios publicados que demuestran que la oseointegración puede lograrse con implantes de carga inmediata, con una reacción tisular comparable a la que se produce en carga diferida ${ }^{109}$. Así, Degidi y cols. ${ }^{68}$ y Neugebauer y cols. ${ }^{94}$ aseguran que la carga inmediata estimula la remodelación y neoformación ósea en la interfase hueso-implante, mientras que Romanos y cols. ${ }^{84}$ añaden que se produce un mayor contacto en esta interfase, incluso en zonas de hueso pobre como afirma Degidi y cols. ${ }^{88}$. En esta misma línea, Nkenke y cols. ${ }^{39}$ confirman que la carga inmediata no afecta la aposición ósea, Meyer ${ }^{40}$ y cols. apuntan que tampoco se afecta la interfase hueso-implante ni la fisiología de los osteoblastos, los cuales sólo necesitan unas horas para adherirse a la superficie del implante según Joos y cols. ${ }^{83}$. Degidi y cols. ${ }^{63.68}$ afirman que tras 6 meses de la carga inmediata hay un $74 \pm 6 \%$ de hueso mineral rodeando el implante en ausencia de gaps, tejido fibroso o infiltrado inflamatorio, del que el $90 \pm 4,5 \%$ era hueso lamelar siendo esta neoformación de tejido óseo lamelar mayor que en carga diferida. Drago y cols. ${ }^{65}$ refieren que la carga inmediata permite conservar la papila, mientras que Lindeboom y cols. ${ }^{96}$ aseguran que no hay diferencias significativas en la pérdida ósea y papilar entre implantes de carga inmediata y diferida, aunque en esta última hay mayor regeneración de la papila mesial.

\section{Conclusiones}

De acuerdo con la revisión efectuada deducimos las conclusiones siguientes:

1. Los factores más determinantes en el éxito de los implantes de carga inmediata son:

- estabilidad primaria

- minimización de micromovimientos

- torque de inserción de al menos $30 \mathrm{Ncm}$

- implantes de superficie rugosa de al menos $10 \mathrm{~mm}$

- oclusión suave, recomendable dieta blanda

- ferulización de los implantes mediante prótesis rígidas

- adecuada cantidad y calidad ósea

2. Está ampliamente demostrado que los resultados obtenidos en rehabilitaciones completas inferiores con implantes de carga inmediata, ya sea mediante sobredentaduras, híbridas o prótesis fijas tipo puente son similares a los conseguidos mediante protocolo convencional. En rehabilitaciones completas maxilares, menos documentadas, los resultados son algo peores, aunque con tasas de éxito también muy altas.

3. En cuanto a rehabilitaciones parciales en maxilar y mandíbula, se consiguen resultados similares en la mayoria de los casos, aunque con menor porcentajes de éxito. Los resultados menos predecibles se producen en restauraciones unitarias y maxilar posterior, por lo que parece lógico actuar con mayor cautela en estos casos.

4. El bruxismo, tabaquismo, diabetes, osteoporosis, síndrome de Sjogrën y pacientes no colaboradores son consideradas situaciones de riesgo relativo para la carga inmediata, mientras que el raquitismo e irradiados de cabeza y cuello suponen una contraindicación absoluta.

5. Histológicamente, está demostrado que puede producirse oseointegración en implantes de carga inmediata, con neoformación de hueso mineral lamelar en la interfase huesoimplante al menos igual que con protocolos clásicos. La pérdida ósea periimplantaria tras el paso de los años también parece ser parecida.

6 La principal ventaja de la carga inmediata es el acortamiento o eliminación del tiempo de espera, lo que supone una gran satisfacción para el paciente y el profesional, aunque a veces hay que asumir riesgos. En función de los casos y el plan de tratamiento, la carga inmediata supondrá mayor, menor o igual coste para el paciente.

7. La carga inmediata es una técnica cada vez más aceptada y predecible que permite la resolución de un creciente número de casos, sin embargo, hay determinadas situaciones clínicas que requieren actuar con cautela a la espera de un mayor número de estudios. 


\section{Bibliografia recomendada}

Para profundizar en la lectura de este tema, el/los autorles considera/an interesantes los artículos que aparecen señalados del siguiente modo: *de interés **de especial interés.

1. Bränemark PI, Hansson BO, Adell R, et al. Intraosseuosanchorage of dental protheses. I. Experimental studies. Scandinavian Journal of Reconstructive Surgery. 1969;3:81-100.

2. Nikolai J, Leslie A. David, George A. Zarb. Carga inmediata de implantes con sobredentaduras inferiors: Resultados clínicos al cabo de un año de un estudio retrospectivo. Revista Internacional de Prótesis Estomatológica. 2006;8:133-140

3. Ledermann PD, Schenk RK, Buser D. Long-lasting osseointegration of immediately loaded, bar-connected TPS screws after 12 years of function: a histologic case report of a 95 year-old patient. Int. Journal Periodontics Restorative Dentistry. 1998;18:552-63

4. Ledermann PD. Principle of the Ledermann screw. Zahnarztl Prax. 1988;8:122-4.

5. Glauser R, Lundgren AK, Gottlow J, Sennerby L, Portmann M, Ruhstaller P, Hammerle CH. Immediate occlusal loading of Branemark TiUnite implants placed predominantly in soft bone: 1-year results of a prospective clinical study. Clin Implant Dent Relat Res. 2003;5:47-56

6. Wolfinger GJ, Balshi TJ, Rangert B. Immediate functional loading of Branemark system implants in edentulous mandibles: clinical report of the results of developmental and simplified protocols. Int J Oral Maxillofac Implants. 2003;18:250-7

7. Calandriello R, Tomatis M, Rangert B. Immediate functional loading of Branemark System implants with enhanced initial stability: a prospective 1- to 2-year clinical and radiographic study. Clin Implant Dent Relat Res. 2003;5:10-20

8. Degidi M, Piattelli A. Immediately loaded barconnected implants with an anodized surface inserted in the anterior mandible in a patient treated with diphosphonates for osteoporosis: a case report with a 12-month follow-up. Clin Implant Dent Relat Res. 2003;5:269-72.

9. Grunder U. Immediate functional loading of immediate implants in edentulous arches: two-year results. Int J Periodontics Restorative Dent. 2001;21:545-51.

10. Ericsson I, Nilson H, Lindh T, Nilner K, Randow. Immediate functional loading of Branemark single tooth implants. An 18 months' clinical pilot follow-up study. Clin Oral Implants Res. 2000;11:26-33.

11. Rocci A, Martignoni M, Burgos PM, Gottlow J, Sennerby L. Histology of retrieved immediately and early loaded oxidized implants: light microscopic observations after 5 to 9 months of loading in the posterior mandible. Clin Implant Dent Relat Res. 2003;5:88-98.

12. Hui E, Chow J, Li D, Liu J, Wat P, Law H.
Immediate provisional for single-tooth implant replacement with Branemark system: preliminary report. Clin Implant Dent Relat Res. 2001;3:79-86.

13. Aparicio Magallón, Carlos, Lundgren, Anna Karin y Rangert, Bo. Carga (función) inmediata vs. carga diferida en implantología: terminología y estado actual. Dientes en el día ${ }^{\circledR}$. RCOE, 2002;7:75-86.

14. Ledermann PD. Stegprothetische Versorgung des zahnlosen Unterkiefers mit Hilfe von plasmabeschichteten Titan-schaubenimplantaten. Dtsch Zahnarztl. 1979;34:907-11.

15. Bränemark P, Hansson B, Adel R, Breine U, Linström J, Hallén $\mathrm{O}$ et al. Osseointegrated implants in the treatment of the edentulous jaw. Experience from a $\mathbf{1 0}$ years period. Scand J Plast Reconstr Surg 1977;16:1-132.

16. Herrera Briones FJ, Romero Olid MN, Vallecilo Capilla M. Update on immediate implant loading: a review of the literature. Med Oral. 2004;9:74-81.

17. Schroeder A, Maeglin B, Sutter F. ITI (Internationales Team fur orale Implantologie) type-F hollow cylinder implant for denture retention in the edentulous jaw. SSO Schweiz Monatsschr Zahnheilkd. 1983;93:720-33.

18. Schroeder A, van der Zypen E, Stich H, Sutter $\mathrm{F}$. The reactions of bone, connective tissue, and epithelium to endosteal implants with titanium-sprayed surfaces. J Maxillofac Surg. 1981;9:15-25.

19. Babbush CA, Kent J, Misiek D. Titanium plasma-sprayed (TPS) screw implants for the reconstruction of the edentulous mandible. J Oral Maxillofacial Surgery 1986;44:274-82.

20. Dietrich U, Skop P, Lippold R, Behnecke N, Wagner W. Vergleich verschiedener Implantatsysteme und deren Prognose im zahnlosen Unterkiefer. Deutsche Zahnärtzliche Zeitung 1993;48:793-6.

21. Chiapasco M, Gatti C, Rossi E, Haefliger W, Markwalder TH. Implant-retained mandibular overdentures with immediate loading. A retrospective multicenter study on 226 consecutive cases. Clin Oral Implant Res 1997;8:48 57.

22. Tarnow DP, Emtiaz S, Classi A. Immediate loading of threaded implants at stage 1 surgery in edentulous arches: Ten consecutive case reports with 1 to 5 years data. Int J Oral Maxillofac Implants 1997;12:319-24

23. Balshi TJ, Wolfinger GJ. Immediate loading of Brånemark implants in edentulous mandibles: A preliminary report. Implant Dent 1997;6:83-8.

24. Scortecci G. Inmediate function of cortically anchored disk-design implants without bone augmentation in moderately to severely resorbed completely edentulous maxillae. J Oral Implant 1999;25:70-9.

25. Horiuchi K, Hiroya U, Yamamoto K, Sugimura M. Immediate loading of Branemark System implants following placement in edentulous patients: a clinical report. Int J Oral Maxillofac Implants 2000;15:824-30.

26. Gatti C, Haefliger W, Chiapasco M. Implantretained mandibular overdentures with immediate loading: a prospective study of ITI implants. Int J Oral Maxillofac Implants 2000; $15: 383-8$.

27. Jaffin RA, Kumar A, Berman CL. Immediate loading of implants in partially and fully edentulous jaws: a series of $\mathbf{2 7}$ cases reports. J Periodontol 2000;71: 833-8.

28. Malo P, Rangert B, Dvarsater L. Immediate function of Branemark implants in the esthetic zone: a retrospective clinical study with 6 months to 4 years of follow-up. Clin Implant Dent Relat Res 2000;2:138-46.

29. Ganeles J, Rosenberg MM, Holt RL, Reichman LH. Immediate loading of implants with fixed restorations in the completely edentulous mandible: report of $\mathbf{2 7}$ patients from a private practice. Int $\mathrm{J}$ Oral Maxillofac Implants 2001; 16:418-26.

30. Glauser R, Ree A, Lundgren A, Gottlow J, Hammerle CH, Scharer P. Immediate occlusal loading of Branemark implants applied in various jawbone regions: a prospective, 1year clinical study. Clin Implant Dent Relat Res 2001; 3:204-13.

31. Ibanez JC, Jalbout ZN. Immediate loading of osseotite implants: 2-year results. Implant Dent 2002; 11:128-36.

32. May D, Romanos GE. Immediated implantsupported mandibular overdentures retained by conical crowns: A new treatment concept. Quintessence International 2002;33:5-12.

33. Pi J, Vericat JA. Branemark Novum ${ }^{\circledR}$ - una alternativa para la rehabilitación del maxilar inferior desdentado. RCOE 2002;7:21-8.

34. Calandriello R, Tomatis M, Rangert B. Immediate functional loading of Branemark System implants with enhanced initial stability: a prospective 1- to 2-year clinical and radiographic study. Clin Implant Dent Relat Res. 2003;5 Suppl 1:10-20.

35. Rocci A, Martignoni M, Gottlow J. Immediate loading in the maxilla using flapless surgery, implants placed in predetermined positions, and prefabricated provisional restorations: a retrospective 3 -year clinical study. Clin Implant Dent Relat Res. 2003;5 Suppl 1:29-36

36. Jivraj SA, Chee WW. An interdisciplinary approach to treatment planning in the esthetic zone. J Calif Dent Assoc. 2003;31:544-9. 
37. Piattelli A, Vrespa G, Petrone G, Iezzi G, Annibali S, Scarano. Role of the microgap between implant and abutment: a retrospective histologic evaluation in monkeys. J Periodontol. 2003;74:346-52

38. Siar CH, Toh CG, Romanos G, Swaminathan D, Ong AH, Yaacob H, Nentwig GH. Periimplant soft tissue integration of immediately loaded implants in the posterior macaque mandible: a histomorphometric study. J Periodontol. 2003;74:571-8.

39. Nkenke E, Lehner B, Weinzierl K, Thams U, Neugebauer J, Steveling H, Radespiel-Troger $\mathrm{M}$, Neukam FW. Bone contact, growth, and density around immediately loaded implants in the mandible of mini pigs. Clin Oral Implants Res. 2003;14:312-21.

40. Meyer U, Wiesmann HP, Fillies T, Joos U. Early tissue reaction at the interface of immediately loaded dental implants. Int J Oral Maxillofac Implants. 2003;18:489-99.

41. Testori T, Del Fabbro M, Szmukler-Moncler S, Francetti L, Weinstein RL. Immediate occlusal loading of Osseotite implants in the completely edentulous mandible. Int J Oral Maxillofac Implants. 2003; 18:544-51.

42. Gapski R, Wang HL, Mascarenhas P, Lang NP. Critical review of immediate implant loading. Clin Oral Implants Res. 2003;14:515-27.

43. Kawahara H, Kawahara D, Hayakawa M, Tamai Y, Kuremoto T, Matsuda S. Osseointegration under immediate loading: biomechanical stress-strain and bone formationresorption. Implant Dent. 2003;12:61-8.

44. Misch CE, Degidi M. Five-year prospective study of immediate/early loading of fixed prostheses in completely edentulous jaws with a bone quality-based implant system. Clin Implant Dent Relat Res. 2003;5:17-28

45. Glauser R, Lundgren AK, Gottlow J, Sennerby L, Portmann M, Ruhstaller P, Hammerle CH. Immediate occlusal loading of Branemark TiUnite implants placed predominantly in soft bone: 1-year results of a prospective clinical study. Clin Implant Dent Relat Res. 2003;5:47-56

46. Morneburg TR, Proschel PA. In vivo forces on implants influenced by occlusal scheme and food consistency. Int J Prosthodont. 2003; 16:481-6.

47. Rocci A, Martignoni M, Gottlow J. Immediate loading of Branemark System TiUnite and machined-surface implants in the posterior mandible: a randomized open-ended clinical trial. Clin Implant Dent Relat Res. 2003;5:5763.

48. Hatano N, Yamaguchi M, Suwa T, Watanabe K. A modified method of immediate loading using Branemark implants in edentulous mandibles. Odontology. 2003;91:37-42.

49. Misch CE, Wang HL. Immediate occlusal loading for fixed prostheses in implant dentistry. Dent Today. $2003 ; 22: 50-6$.

50. Degidi M, Scarano A, Petrone G, Piattelli A. Histologic analysis of clinically retrieved immediately loaded titanium implants: a report of 11 cases. Clin Implant Dent Relat Res. 2003;5:89-93.

51. Engstrand P, Grondahl K, Ohrnell LO, Nilsson P, Nannmark U, Branemark PI. Prospective follow-up study of 95 patients with edentulous mandibles treated according to the Branemark Novum concept. Clin Implant Dent Relat Res. 2003;5:3-10.

52*. Malo P, Rangert B, Nobre M. «All-on-Four» immediate-function concept with Branemark System implants for completely edentulous mandibles: a retrospective clinical study. Clin Implant Dent Relat Res. 2003;5:29.

53. Malo P, Friberg B, Polizzi G, Gualini F, Vighagen $T$, Rangert B. Immediate and early function of Branemark System implants placed in the esthetic zone: a 1-year prospective clinical multicenter study. Clin Implant Dent Relat Res. 2003;5:37-46.

54. Vanden Bogaerde L, Pedretti G, Dellacasa P, Mozzati M, Rangert B, Wendelhag I. Early function of splinted implants in maxillas and posterior mandibles, using Branemark System Tiunite implants: an 18-month prospective clinical multicenter study. Clin Implant Dent Relat Res. 2004;6:121-9

55. Romanos GE. Surgical and prosthetic concepts for predictable immediate loading of oral implants. J Calif Dent Assoc. 2004;32: 991-1001.

56. Olsen S, Ferguson SJ, Sigrist C, Fritz WR, Nolte LP, Hallermann W, Caversaccio M. A novel computational method for real-time preoperative assessment of primary dental implant stability. Clin Oral Implants Res. 2005 Feb;16: 53-9.

57. Cochran DL, Morton D, Weber HP. Consensus statements and recommended clinical procedures regarding loading protocols for endosseous dental implants. Int J Oral Maxillofac Implants. 2004;19:109-13.

58. Morton D, Jaffin R, Weber HP. Immediate restoration and loading of dental implants: clinical considerations and protocols. Int $\mathrm{J}$ Oral Maxillofac Implants. 2004;19:103-8.

59. Ganeles J, Wismeijer D. Early and immediately restored and loaded dental implants for single-tooth and partial-arch applications. Int J Oral Maxillofac Implants. 2004;19:92-102.

60. Uribe R, Penarrocha M, Balaguer J, Fulgueiras N. Immediate loading in oral implants. Present situation. Med Oral Patol Oral Cir Bucal. 2005; 10:143-53.

61. Misch CE, Wang HL, Misch CM, Sharawy M, Lemons J, Judy KW. Rationale for the application of immediate load in implant dentistry: part II. Implant Dent. 2004 ; 13:310-21.

62. Testori T, Del Fabbro M, Galli F, Francetti L, Taschieri S, Weinstein R. Immediate occlusal loading the same day or the after implant placement: comparison of 2 different time frames in total edentulous lower jaws. J Oral Implantol. 2004;30:307-13.

63*. Degidi M, Scarano A, Piattelli M, Piattelli A. Histologic evaluation of an immediately loa- ded titanium implant retrieved from a human after 6 months in function. J Oral Implantol. 2004;30:289-96.

64**. Jaffin RA, Kumar A, Berman CL. Immediate loading of dental implants in the completely edentulous maxilla: a clinical report. Int J Oral Maxillofac Implants. 2004;19:72130 .

65. Drago CJ, Lazzara RJ. Immediate provisional restoration of Osseotite implants: a clinical report of 18-month results. Int J Oral Maxillofac Implants. 2004;19:534-41

66. Gallucci GO, Bernard JP, Bertosa M, Belser UC. Immediate loading with fixed screwretained provisional restorations in edentulous jaws: the pickup technique. Int J Oral Maxillofac Implants. 2004;19:524-33.

67. Castellon P, Block MS, Smith MB, Finger IM Immediate loading of the edentulous mandible: delivery of the final restoration or a provisional restoration--which method to use?. J Oral Maxillofac Surg. 2004;62:30-40.

68. Degidi M, Scarano A, Piattelli M, Perrotti V, Piattelli A. Bone remodeling in immediately loaded and unloaded titanium dental implants: a histologic and histomorphometric study in humans. J Oral Implantol. 2005; 31:18-24.

69. Martínez JM, Barona C, Cano J, Fernández F, Sánchez A. Evaluación de 80 implantes, sometidos a carga inmediata en desdentados inferiores tras un seguimiento de dos años. Med. Oral Patol. Oral Cir. Bucal 2006;11:16570 .

70. Balshi SF, Wolfinger GJ, Balshi TJ. A prospective study of immediate functional loading, following the Teeth in a Day protocol: a case series of 55 consecutive edentulous maxillas. Clin Implant Dent Relat Res. 2005;7:24-31.

71. Aalam AA, Nowzari H, Krivitsky A. Functional restoration of implants on the day of surgical placement in the fully edentulous mandible: a case series. Clin Implant Dent Relat Res. 2005;7:10-6.

72. Tsirlis AT. Clinical evaluation of immediate loaded upper anterior single implants. Implant Dent. 2005;14:94-103.

73. Degidi M, Piattelli A. 7-year follow-up of $\mathbf{9 3}$ immediately loaded titanium dental implants. J Oral Implantol. 2005;31:25-31.

74. Nkenke E, Lehner B, Fenner M, Roman FS, Thams U, Neukam FW, Radespiel-Troger M. Immediate versus delayed loading of dental implants in the maxillae of minipigs: followup of implant stability and implant failures. Int J Oral Maxillofac Implants. 2005;20:39-47.

75. Abboud M, Koeck B, Stark H, Wahl G, Paillon $\mathrm{R}$. Immediate loading of single-tooth implants in the posterior region. Int $\mathrm{J}$ Oral Maxillofac Implants. 2005;20:61-8.

76*. Ibanez JC, Tahhan MJ, Zamar JA, Menendez AB, Juaneda AM, Zamar NJ, Monqaut JL. Immediate occlusal loading of double acidetched surface titanium implants in 41 consecutive full-arch cases in the mandible and 
maxilla: 6- to 74-month results. J Periodontol. 2005;76:1972-81.

77*. Degidi M, Piattelli A, Felice P, Carinci F Immediate functional loading of edentulous maxilla: a 5-year retrospective study of $\mathbf{3 8 8}$ titanium implants. J Periodontol. 2005;76: 1016-24.

78. Weischer T, Kandt M, Reidick T. Immediate loading of mandibular implants in compromised patients: preliminary results. Int $J$ Periodontics Restorative Dent. 2005;25:501-7.

79. Jivraj S, Reshad M, Chee WW. Critical appraisal. Immediate loading of implants in the esthetic zone. J Esthet Restor Dent. 2005; 17:320-5.

80. Perez del Palomar A, Arruga A, Cegonino J, Doblare M. A finite element comparison between the mechanical behaviour of rigid and resilient oral implants with respect to immediate loading. Comput Methods Biomech Biomed Engin. 2005;8:45-57.

81. Attard NJ, Zarb GA. Immediate and early implant loading protocols: a literature review of clinical studies. J Prosthet Dent 2005; $94: 242-58$

82. Ioannidou E, Doufexi A. Does loading time affect implant survival? A meta-analysis of 1.266 implants. J Periodontol. 2005;76:1252-8

83. Joos U, Buchter A, Wiesmann HP, Meyer U. Strain driven fast osseointegration of implants. Head Face Med. 2005;1:6.

84. Romanos GE, Testori T, Degidi M, Piattelli A. Histologic and histomorphometric findings from retrieved, immediately occlusally loaded implants in humans. J Periodontol. 2005; 76:1823-32. Erratum in: J Periodontol. 2006 Feb;77:326.

85. Albrektsson T, Wennerberg A. Oral implan surfaces: Part 1-review focusing on topographic and chemical properties of different surfaces and in vivo responses to them. Int J Prosthodont. 2004;17:536-43.

86. Albrektsson T, Wennerberg A. Oral implant surfaces: Part 2--review focusing on clinical knowledge of different surfaces. Int J Prosthodont. 2004; 17:544-64.

87. Attard NJ, David LA, Zarb GA. Immediate loading of implants with mandibular overdentures: one-year clinical results of a prospective study. Int J Prosthodont. 2005;18:463-70.

88. Degidi M, Scarano A, Iezzi G, Piattelli A. His- tologic analysis of an immediately loaded implant retrieved after 2 months. J Oral Implantol. 2005;31:247-54

89. Ostman PO, Hellman M, Sennerby L. Direct implant loading in the edentulous maxilla using a bone density-adapted surgical protocol and primary implant stability criteria for inclusion. Clin Implant Dent Relat Res. 2005; 7:S60-9.

90. Attard NJ, Laporte A, Locker D, Zarb GA. A prospective study on immediate loading of implants with mandibular overdentures: patient-mediated and economic outcomes. Int J Prosthodont. 2006;19:67-73.

91. Duyck J, Vandamme K, Geris L, Van Oosterwyck H, De Cooman M, Vandersloten J, Puers R, Naert I. The influence of micromotion on the tissue differentiation around immediately loaded cylindrical turned titanium implants. Arch Oral Biol. 2006;51:1-9.

92. Yoo RH, Chuang SK, Erakat MS, Weed M, Dodson TB. Changes in crestal bone levels for immediately loaded implants. Int J Oral Maxillofac Implants. 2006;21:253-61.

93. Ormianer Z, Garg AK, Palti A. Immediate loading of implant overdentures using modified loading protocol. Implant Dent. 2006;15:3540.

94. Neugebauer J, Traini T, Thams U, Piattelli A, Zoller JE. Peri-implant bone organization under immediate loading state. Circularly polarized light analyses: a minipig study. J Periodontol. 2006;77:152-60.

95. Drago CJ, Lazzara RJ. Immediate occlusal loading of Osseotite implants in mandibular edentulous patients: a prospective observational report with 18-month data. J Prosthodont. 2006; 15:187-94.

96. Lindeboom JA, Frenken JW, Dubois L, Frank M, Abbink I, Kroon FH. Immediate loading versus immediate provisionalization of maxillary single-tooth replacements: a prospective randomized study with BioComp implants. J Oral Maxillofac Surg. 2006;64:936-42.

97. Sul YT, Johansson C, Albrektsson T. Which surface properties enhance bone response to implants? Comparison of oxidized magnesium, TiUnite, and Osseotite implant surfaces. Int J Prosthodont. 2006;19:319-28.

98. Le Guehennec L, Soueidan A, Layrolle P, Amouriq Y. Surface treatments of titanium dental implants for rapid osseointegration Dent Mater. 2006;10

99*. Degidi M, Piattelli A, Carinci F. Parallel screw cylinder implants: comparative analysis between immediate loading and two-stage healing of 1,005 dental implants with a 2-year follow up. Clin Implant Dent Relat Res. 2006;8:151-60

100. Klee de Vasconcellos D, Bottino MA, Saad PA Faloppa F. A new device in immediately loaded implant treatment in the edentulous mandible. Int $\mathrm{J}$ Oral Maxillofac Implants. 2006;21:615-22

101. Sul YT, Jeong Y, Johansson C, Albrektsson T Oxidized, bioactive implants are rapidly and strongly integrated in bone. Part 1 - experimental implants. Clin Oral Implants Res. 2006; 17:521-6.

102. Nkenke E, Fenner M. Indications for immediate loading of implants and implant success. Clin Oral Implants Res. 2006;17:19-34.

103**. Davies, J.E. Mecanismos de integración endoósea. Revista Internacional de Prótesis Estomatológica. 1999;1.

104*. Lekholm U. Carga inmediata y temprana en los implantes dentales en pacientes de riesgo. Periodontol 2000. 2003;33:194-203.

105. Isidor $F$. Influence of forces on peri-implant bone. Clin Oral Implants Res. 2006;17:8-18.

106*. Albrektsson T, Zarb G, Worthington P, Eriksson AR. The long-term efficacy of currently used dental implants: a review and proposed criteria of success. Int $J$ Oral Maxillofac Implants. 1986;1:11-25.

107. Albrektsson T, Sennerby L. Direct bone anchorage of oral implants: clinical and experimental considerations of the concept of osseointegration. Parodontol. 1990;1:307-20.

108. Calandriello R. Tomatis M. Immediate function of single implants using Bränemark System: prospective one year report of final restorations. 2004;4:32-40.

109. Glauser R, Zembic A, Hammerle CH. A systematic review of marginal soft tissue at implants subjected to immediate loading or immediate restoration. Clin Oral Implants Res. 2006;17:82-92.

110. O'Sullivan D, Sennerby L, Jagger D, Meredith N. A comparison of two methods of enhancing implant primary stability. Clin Implant Dent Relat Res. 2004;6:48-57. 